\title{
Corpus
}

\section{Un hommage à Thierry Chanier}

\section{Céline Poudat}

\section{(2) OpenEdition \\ Journals}

\section{Electronic version}

URL: http://journals.openedition.org/corpus/4731

DOI: 10.4000/corpus.4731

ISSN: $1765-3126$

\section{Publisher}

Bases; corpus et langage - UMR 6039

\section{Electronic reference}

Céline Poudat, «Un hommage à Thierry Chanier », Corpus [Online], 20 | 2020, Online since 21 January 2020, connection on 08 September 2020. URL : http://journals.openedition.org/corpus/4731 ; DOI : https://doi.org/10.4000/corpus.4731

This text was automatically generated on 8 September 2020

(c) Tous droits réservés 


\title{
Un hommage à Thierry Chanier
}

\author{
Céline Poudat
}

1 Nous dédions ce numéro sur les corpus complexes à notre collègue Thierry Chanier, qui a joué un rôle majeur dans le consortium Corpus Écrits (2011-2015) en mettant notamment en œuvre le projet CoMeRe qui fut l'une des plus grandes réussites du consortium, et qui a cessé ses activités académiques depuis mars 2017.

2 Revenons d'abord en deux mots sur le parcours de Thierry Chanier, qui fut l'un des premiers en France à développer les liens entre Intelligence Artificielle (IA) et Traitement Automatique des Langues (TAL).

3 Le parcours de Thierry est d'abord fortement marqué par l'idée d'engagement. Engagement politique d'abord, puisque Thierry démarra ses jeunes années comme militant, et engagement scientifique ensuite, lorsqu'il découvre l'intelligence artificielle au détour d'une rencontre avec Michel Chambreuil, l'un des premiers sémanticiens à avoir travaillé sur la grammaire de Montague.

4 Thierry avait entre-temps repris des études classiques d'informatique à ClermontFerrand mais se sentait déjà accablé par la carrière d'ingénieur en informatique de gestion qui l'attendait et qui avait justifié son financement régional. Chambreuil lui apporta donc un véritable élan: ils mirent en place un groupe autour de l'IA à Clermont, centré sur l'ingénierie cognitive de formation, et Chambreuil fit entrer Thierry à Bull, grande entreprise française d'informatique de l'époque, en 1986. Thierry réalisa d'abord un stage sur la question de l'IA appliquée à l'apprentissage, et poursuivit une thèse sur le traitement automatique de textes à caractère technique à Paris 13 sous la direction de Daniel Kaiser, un ancien de l'IA en France.

5 À la suite de sa thèse, Thierry chercha un post-doctorat à l'étranger, qu'il effectuera en Grande-Bretagne à Lancaster University, toujours dans le domaine de l'IA appliquée à l'éducation et au langage, et plus spécifiquement, à l'apprentissage des langues. Il débusqua ce post-doc dans un colloque international à Montréal Intelligence tutoring systems, muni de la liste des conférenciers invités et de son CV, ce qui montre bien sa pugnacité. John Self fut le premier conquis par Thierry et l'invita à donner un séminaire dans son laboratoire d'informatique (Université de Lancaster). C'est dans le cadre de ce post-doctorat que Thierry développe son futur réseau CALL (Computer- 
Assisted Language Learning) au travers de chercheurs de différents pays qu'il rencontre dans un séminaire de l'OTAN à Washington.

Thierry devient ensuite maître de conférences à Paris 5 de 1990 à 1995. Il collabore avec Desclés, qui développe un réseau des sciences cognitives dans les années 1990 que Thierry intègre, et ils montent ensemble une option de DEA ${ }^{1}$.

7 En parallèle, Thierry continue de développer sa recherche à Clermont-Ferrand dans un département de linguistique de la faculté des lettres: il crée le Laboratoire de Recherche sur le Langage (LRL) avec Chambreuil en 1991, et il monte et décroche le projet européen Camille en 1993. C'est dans ce cadre que sont développés les premiers logiciels (CD-ROM) d'aide à l'apprentissage du Français Langue Étrangère (FLE) (le second logiciel sera développé en 1995), en collaboration avec des collègues espagnols, hollandais et anglais. Camille sera parmi les premiers (le deuxième si l'on veut être précis) logiciel multimédia français d'aide à l'apprentissage du FLE.

8 Thierry contribue ainsi au développement de l'ALMT (Apprentissage des langues médié par les technologies) et passe son HDR en 1995 à l'Université Blaise Pascal (Clermont 2) sur ces aspects.

En 1996, il obtient un poste de professeur à Besançon, toujours en faculté des lettres. C'est un poste d'informatique appliquée aux sciences humaines. Thierry rejoint le Laboratoire d'informatique qui poursuit d'autres thématiques, mais le laisse développer ses travaux.

10 En 1998, il crée avec Françoise Demaizière et Maguy Pothier, la revue Alsic (Apprentissage des Langues et Systèmes d'Information et de Communication), qui fut parmi les premières revues universitaires en ligne et en accès ouvert avec un comité de relecture, ce qui était chose rare à l'époque. Alsic permet de structurer le domaine et Thierry contribue à intégrer la communauté francophone dans la sphère européenne en obtenant l'organisation de la conférence EUROCALL (European Association for ComputerAssisted Language Learning) en France pour la première fois. EUROCALL se tient donc à Besançon en 1999.

11 C'est également à Besançon que Thierry monte différents projets européens, de même qu'une ANR, Mulce, sur la structuration des données. C'était la première fois que l'ANR s'intéressait explicitement aux corpus déjà constitués, qui devaient donc être structurés et organisés pour pouvoir être échangés. On voit aujourd'hui à quel point cette idée est intégrée, et c'est d'ailleurs dans cet esprit que nous œuvrons dans le consortium de linguistique CORLI${ }^{2}$, avec des appels annuels à finalisation de corpus. Profitons-en également pour mentionner l'ouvrage de Thierry paru en $2004 \mathrm{chez}$ l'Harmattan et librement accessible depuis HAL, Archives ouvertes et publication scientifique. Comment mettre en place l'accès libre aux résultats de la recherche? Cet ouvrage a été écrit durant la mission que lui avait confiée le ministère de la recherche pour développer les archives ouvertes en sciences humaines (d'abord l'archive Edutice, qui inspira HAL-SHS à ses débuts).

12 En 2009, Thierry, qui est resté très attaché à Clermont-Ferrand, y obtient sa mutation. Il prend alors la direction du LRL (Laboratoire de Recherche sur le Langage) et participe au projet européen Archi21 sur l'apprentissage des langues dans les mondes synthétiques, qui intègre l'apprentissage de l'architecture et l'apprentissage des langues étrangères. Il fédère autour de lui une équipe de doctorants qui le reconnaît tant pour sa disponibilité en tant qu'encadrant que par son obsession du rangement. 
13 En 2011, il croise la route des consortiums Corpus, et l'expérience de Thierry sur les corpus complexes et les standards nous a été extrêmement profitable. Il s'est ainsi particulièrement impliqué dans l'IR Corpus écrits d'abord, dans le cadre du GT7 nouvcom que nous avons coordonné ensemble, puis dans le cadre du consortium CORLI, avec le groupe MultiCom que nous avons continué à copiloter ensemble avant qu'il ne prenne sa retraite. De ce GT7 naquit le projet national CoMeRe, que nous avons eu le plaisir de présenter ensemble dans ce numéro, Ciara Wigham et moi.

14 Fort de son expérience CALL, Thierry a entrepris avec son efficacité habituelle une réflexion active sur les corpus CMC, qu'il appréhendait depuis 2004-2005 dans le contexte de l'apprentissage (voir l'article d'Eva Lacroix dans ce numéro). Il a dirigé le projet de manière particulièrement diligente et efficace en collaborant avec les acteurs européens, dont Michael Beisswenger, sur la question des standards et de l'annotation TEI-CMC. Michael Beisswenger et Harald Lüngen présentent d'ailleurs le CMC-Core dans notre numéro.

15 Aujourd'hui, Thierry démarre (encore!) une autre vie: après la politique et le militantisme, après CALL, après la CMR, il s'attaque maintenant à la haute montagne. Depuis une dizaine d'années, il a commencé de s'impliquer davantage dans le club Alpin français dont il était membre (et on sait ce que s'impliquer veut dire pour Thierry !). Il fait de la formation et prépare actuellement une formation de secours en montagne - il recycle en fait ses compétences en gestion de projet.

16 En parallèle puisqu'il s'agit après tout d'une seconde vie qu'il démarre, il a aussi été pendant deux ans Délégué de l'éducation nationale, c'est-à-dire qu'il a été volontaire bénévole dans les écoles primaires ou maternelles. C'est un travail de médiation entre le corps des enseignants de l'école, la municipalité et les représentants de parents d'élèves. Il assistait aux conseils d'école, faisait un bilan annuel de l'école dont il s'occupait, tout en montant des dossiers pour que l'école soit notamment équipée en tableaux blancs interactifs.

17 Nous sommes donc extrêmement heureux de lui rendre aujourd'hui hommage par ce numéro, que nous espérons tous qu'il appréciera autant que nous avons pris plaisir à le préparer.

\section{NOTES}

1. Diplôme d'Études Approfondies, qui correspond aujourd'hui à une deuxième année de Master.

2. https://corli.huma-num.fr/ 